\title{
Le jeu des positions de savoir en recherche collaborative : une analyse des points de vue négociés d'un groupe d'enseignantes du primaire ${ }^{1}$
}

Joëlle Morrissette, Ph.D.

Université de Montréal

Serge Desgagné, Ph.D.

Université Laval

\begin{abstract}
Résumé
Cet article offre un éclairage, inspiré d'une perspective interactionniste, sur la négociation de sens par laquelle les partenaires d'une approche collaborative ajustent leurs positions quant au savoir à construire, sur la base de la proposition de complémentarité que leur propose une chercheuse. Cet éclairage s'appuie sur une analyse de discours issue d'entretiens menés avec un groupe de cinq enseignantes du primaire dans le cadre d'une recherche doctorale portant sur les pratiques d'évaluation formative des apprentissages. L'analyse conduit à esquisser un portrait des positions de savoir des participantes, de leur façon d'en négocier une viabilité dans le cadre de l'offre de complémentarité souhaitée; à titre d'illustration, quelques moments-types où se négocient les positions de savoir en cours de démarche sont présentés. Il ressort de cette analyse que la démarche de coconstruction de savoir fait l'objet d'une négociation continue dans l'interaction entre partenaires de la recherche.
\end{abstract}

Mots clés

RECHERCHE COLLABORATIVE, INTERACTIONNISME SYMBOLIQUE, ANALYSE DE DISCOURS, ÉVALUATION FORMATIVE, ENSEIGNEMENT PRIMAIRE.

\section{Introduction}

L'approche de recherche collaborative en éducation s'est pour beaucoup développée selon une visée de rapprochement entre deux mondes parfois

RECHERCHES QUALITATIVES - Vol. 28(2), 2009, pp. 118-144.

APPROCHES INDUCTIVES II

ISSN 1715-8702 - http://www.recherche-qualitative.qc.ca/Revue.html

(C) 2009 Association pour la recherche qualitative 
difficiles à concilier : celui des chercheurs et celui des praticiens (Desgagné, 1997, 2001, 2007; Desgagné, Bednarz, Couture, Poirier \& Lebuis, 2001). Est sous-jacente à cette visée de médiation entre ces deux mondes, la reconnaissance de deux logiques de penser et d'agir, associées d'une part aux enjeux respectifs de la communauté scientifique qui guident les intérêts des chercheurs et, d'autre part, à la communauté professionnelle qui guident les intérêts des praticiens. À l'horizon de cette visée de rencontre, la coconstruction d'un savoir professionnel qui soit le produit combiné et inédit des logiques, intérêts et enjeux des uns et des autres. C'est là l'expression de l'arrimage souhaité entre la théorie et la pratique pour un savoir professionnel qui puisse être reconnu tant par la communauté scientifique que par la communauté des praticiens.

Penser d'une telle manière la collaboration de recherche pourrait laisser croire que le rapprochement de ces deux logiques de penser et d'agir va de soi, du moment qu'on réunit chercheurs et praticiens dans un même «espace réflexif » en vue d'assumer leur démarche de coconstruction de savoir. Rien n'assure pourtant que les logiques des uns et des autres pour penser le savoir soient compatibles; si elles le sont, on peut penser qu'en cours de démarche, les partenaires auront néanmoins à négocier entre eux leur manière d'envisager le savoir à coconstruire. Surtout qu'à l'intérieur même d'une logique pour penser le savoir, chaque acteur, chercheur ou praticien, va se donner une représentation non seulement de sa propre contribution, mais aussi de la contribution de l'autre. Autrement dit, dans une perspective de collaboration, s'inscrire dans une logique pour concevoir le savoir à coconstruire, c'est d'emblée penser la complémentarité avec ses vis-à-vis partenaires.

C'est un peu dans cet esprit d'éclairer la part de négociation entre les partenaires autour du savoir à coconstruire dans une recherche collaborative que nous avons été amenés à proposer, à l'appui d'une perspective interactionniste, une analyse de ce que nous avons appelée «le jeu des positions de savoir ». Comme on le verra, c'est à partir d'une recherche portant sur les pratiques d'évaluation formative des apprentissages qu'est élaborée cette analyse.

\section{Le contexte de la recherche qui sert d'ancrage à l'analyse}

La problématique liée à notre proposition d'analyse s'ancre à la recherche doctorale de Morrissette (2009) qui visait à documenter le savoir-faire mis en œuvre par des enseignantes du primaire en matière d'évaluation formative des apprentissages, et ce, à partir de l'explicitation, en groupe, de leurs « manières de faire " (de Certeau, 1990). La pertinence sociale de ce projet est mise en relief par le contexte d'une réflexion qui a cours dans la plupart des pays 
occidentaux autour de la réussite éducative. En effet, durant la dernière décennie, on a vu se multiplier les réformes des systèmes éducatifs qui visent à amener un plus grand nombre d'élèves à franchir le processus de scolarisation avec succès. Ce contexte particulier contribue à mettre au cœur des préoccupations l'évaluation formative des apprentissages, c'est-à-dire un processus d'évaluation continu mis en œuvre par les enseignants qui prend la forme d'un soutien explicite à l'apprentissage plutôt que d'une sanction. Plusieurs auteurs (par exemple, Allal, 1991; Perrenoud, 1998; Scallon, 2000) soutiennent d'ailleurs que ce type d'évaluation est d'une grande importance en raison des possibilités d'amélioration et de correction des processus d'apprentissage qu'il favorise; dit autrement, l'évaluation formative serait au cœur de la réussite des élèves.

Toutefois, sur la base d'une recension d'écrits et de travaux antérieurs (Morrissette, 2002; Morrissette \& Maheux, 2007), il semble que jusqu'à présent, c'est dans une perspective normative et prescriptive que les pratiques d'évaluation formative ont été étudiées, tant et si bien que la recherche émet des propositions sur «ce qui devrait se passer", mais demeure muette relativement à « ce qui se passe » sur le terrain de la pratique enseignante. En s'appuyant sur des recherches plus éloquentes telles que celles de Bell et Cowie (2001) ou d'Elliott (1999), il paraît intéressant d'aborder ces pratiques partagées par les enseignants sur une base quotidienne sous un angle plus compréhensif. En ce sens, la recherche à partir de laquelle la présente analyse est tirée a visé à documenter les manières de faire l'évaluation formative sur la base de l'explicitation qu'en font leurs maîtres d'œuvre, soit les enseignants considérés comme des "acteurs compétents » au sens où l'entend Giddens (1987), c'est-à-dire qui jouissent d'une marge de manœuvre, disposent de ressources pour agir, réfléchissent sur cet agir et peuvent le théoriser ou le rationaliser lorsque sollicités, comme argumenté précédemment (Guignon \& Morrissette, 2006).

De manière concrète, une collaboration de recherche a été établie avec un groupe d'enseignantes du primaire qui se sont engagées dans une démarche d'explicitation de leurs manières de faire l'évaluation formative. Cinq enseignantes d'une même école de la région de Québec, oeuvrant en $4^{\mathrm{e}}, 5^{\mathrm{e}}$ et $6^{\mathrm{e}}$ année, ont été invitées à collaborer à la recherche entre juin et décembre 2006. Le format d'investigation a reposé sur un dispositif composé de trois types d'activités réflexives complémentaires proposés en alternance, et cette microanalyse s'attarde à l'une de ces activités, soit la série d'entretiens de groupe qui a ponctué l'ensemble du projet ${ }^{2}$. Dans le cadre de cette activité soutenue par l'animation de la chercheuse, les enseignantes ont partagé leurs réflexions, débattu de leurs pratiques respectives en tentant de se situer les unes par 
rapport aux autres, voire en trouvant, à certains moments, un terrain d'entente, une zone partagée de sens autour de l'évaluation formative.

L'intérêt porté à ces entretiens de groupe réside dans le fait qu'en tant qu'espaces délibératifs, ils représentent le moyen le plus susceptible, par la forme de débat qu'ils proposent entre les partenaires, de mettre en lumière le jeu des "positions » ${ }^{3}$ devant le savoir à coconstruire. En effet, en débattant collectivement de leurs manières respectives de faire l'évaluation formative et en justifiant ce qui en fonde la validité pour elles-mêmes, les enseignantes se sont inscrites dans un certain rapport de complémentarité avec la chercheuse. Et ce rapport suggère que la chercheuse s'est placée en facilitatrice du partage des pratiques et du débat qui pouvait naître autour de leur explicitation. Un questionnement émerge de ce rapport de complémentarité à établir entre les partenaires. De quelle manière ces enseignantes ont-elles répondu à cette proposition de complémentarité? Ont-elles accepté la «position » que leur suggérait la chercheuse devant le savoir à coconstruire? C'est ce que notre micro-analyse des entretiens de groupe tente d'éclairer.

\section{La contribution de cette analyse à l'approche collaborative}

Jusqu'à présent, les travaux sur l'approche collaborative qui ont cherché à éclairer ce qui se passe dans cet «espace réflexif " se sont plus attardés à documenter la double contribution de la logique théorique et de la logique pratique dans le savoir professionnel à coconstruire, entre autres, en tentant de distinguer les cadres de référence respectifs des chercheurs et des praticiens dans la démarche de recherche (voir notamment Bednarz, Desgagné, Diallo \& Poirier, 2001, ainsi que Couture, 2002). Mais ces travaux, en choisissant cet angle de la double contribution au savoir coconstruit, ont laissé à l'arrière-plan la négociation ou l'ajustement de la complémentarité attendue entre les partenaires dans la construction du savoir. Comment se négocie l'offre d'un chercheur d'un rapport égalitariste, non prescriptif, dans le cadre de laquelle le point de vue réflexif des praticiens est sollicité?

Cet angle inédit pour documenter ce qui se passe dans cet «espace réflexif » que constituent les entretiens de groupe nous conduit à aborder la collaboration comme une négociation de «positions" devant le savoir à construire de la part des acteurs qui interagissent entre eux. En effet, pour Darré (1999), qui développe cette manière d'aborder la collaboration, ce qui se joue entre le chercheur et le praticien, ce n'est pas tant la question qu'ils possèdent des savoirs différents qu'il faudrait mettre à contribution, dans un projet collaboratif, que la question du statut qu'ils accordent au savoir de l'autre, s'ils lui en accordent un, de même qu'à leur propre savoir. Ainsi, un peu en corollaire à l'opposition que fait Schön $(1983,1987)$ entre rationalité 
technique et rationalité pratique, Darré (1999) dénonce les positions traditionnelles entretenues entre chercheurs et praticiens, les premiers recevant le statut de "concepteurs » du savoir, les seconds, d' " exécutants ». Pour le chercheur qui souhaite un rapport plus égalitaire, la question devient alors de convaincre les praticiens qu'il ne se présente pas lui-même en détenteur du savoir, et qu'il sollicite leur participation à titre de producteurs du savoir. En somme, le défi devient alors, pour le chercheur, de modifier sensiblement le rapport dissymétrique entre les partenaires, de modifier les « positions » devant le savoir ${ }^{4}$.

Darré (1999) propose en ce sens de créer des dispositifs de recherche, comme cet «espace réflexif » que suggère l'approche collaborative, qui vont faciliter la mise en place de ce nouveau rapport. Mais on peut bien penser qu'il ne suffit pas, pour le chercheur, d'en être convaincu pour que cela se produise. Si l' « espace réflexif » se veut un lieu symbolique d'élaboration d'un nouveau savoir au carrefour des logiques pratique et théorique assumées par les partenaires, il est aussi un lieu symbolique de négociation des «positions » de savoir tenues par ces mêmes partenaires, négociation qui va dans le sens d'une symétrisation des «positions ». Dans cette perspective, le chercheur va jouer un rôle important dans le maintien d'un certain équilibre en relation avec le nouveau rapport à établir au savoir, soit la nouvelle complémentarité à créer entre les partenaires, de sorte que les praticiens puissent prendre leur place d'acteurs réflexifs et donc de contributeurs à la production du savoir. Qu'en est-il dans la recherche qui nous occupe? Comment se sont négociées, lors des entretiens collectifs, les "positions » devant le savoir à construire sur la base de la proposition de complémentarité proposée par la chercheuse? C’est là la perspective originale par laquelle nous tenterons d'éclairer la démarche collaborative dans le projet concerné.

\section{L'interactionniste symbolique comme cadre d'analyse}

Choisir d'investiguer la collaboration sous cet angle des positions de savoir revendiquées par les partenaires, c'est s'inscrire de plain-pied dans la tradition de l'interactionnisme symbolique (Becker \& McCall, 1990; Woods, 1992). En effet, en s'appuyant sur cette perspective, on peut concevoir que le rapport au savoir qu'on projette de coconstruire est le fruit d'interactions symboliques, soit du sens que l'un et l'autre partenaires de l'interaction se renvoient réciproquement à propos de ce qui fait l'objet de leur construction de sens. En d'autres mots, ce savoir serait tributaire de la manière dont chacun attribue à lui-même et aux autres une certaine contribution attendue dans la démarche de coconstruction. Et c'est bien cela qui nous intéresse ici, soit ce jeu des positions de savoir qui se négocient dans l'interaction entre les acteurs en présence. 
La métaphore théâtrale de Goffman (1973), un sociologue s'étant particulièrement intéressé aux situations d'interaction de face-à-face, est instructive pour éclairer le jeu des positions de savoir qui s'est négocié dans l'espace réflexif proposé aux enseignantes. En effet, l'auteur envisage la vie sociale comme une scène sur laquelle des acteurs en représentation construisent une "définition commune de la situation ». Chacun se met en scène en prenant part activement à l'action, offrant ainsi aux autres l'image qu'ils se donnent, une représentation qu'ils ont d'eux-mêmes (le self), le rôle qu'ils ont décidé d'adopter en fonction de leur interprétation de la situation qui se présente à eux. En s'appuyant sur cette métaphore, on peut concevoir qu'au cours des activités réflexives qui ont eu lieu lors des entretiens, les enseignantes se sont mises en scène afin de contribuer au projet collectif. Plus spécifiquement, elles auraient mis en scène dans la dynamique de l'interaction leur représentation de la coconstruction des savoirs, c'est-à-dire leur façon d'envisager la complémentarité entre chercheur et praticiens.

Il ne faudrait pas imaginer leur façon de se représenter ce face-à-face comme étant quelque chose de figé, de statique. En effet, comme le soutient Becker (1986, 2004), une des figures de proue de l'interactionnisme symbolique, il convient d'envisager que les acteurs s'ajustent constamment aux autres, redéfinissant constamment la situation dans laquelle ils sont impliqués, adaptant leurs stratégies d'action en tenant compte des réponses potentielles de toutes les personnes qui sont aussi impliquées. Comme il le précise, les acteurs s'ajustent constamment aux autres, redéfinissant constamment la situation dans laquelle ils sont impliqués, adaptant leurs stratégies d'action en tenant compte des réponses potentielles de toutes les personnes qui sont aussi impliquées. Becker parle de cette dynamique en termes de «coopération ", non pas dans le sens moral du terme, mais bien dans le sens d'une forme d'interdépendance qui permet d'interagir de façon relativement satisfaisante, dans la poursuite d'un projet commun. Cette invitation à penser en termes plus dynamiques la rencontre entre une chercheuse et des praticiennes amène à considérer que la représentation initiale que chaque acteur se faisait de la complémentarité dans la coconstruction du savoir est l'objet d'une négociation dans l'interaction, une négociation notamment influencée par le rapport plus égalitariste proposé par la chercheuse qui sollicite le point de vue réflexif des praticiennes. En réponse à l'invitation qui a été faite aux enseignantes, soit d'entrer dans une démarche d'explicitation de leurs manières de faire l'évaluation formative, il s'agit donc de comprendre comment les enseignantes ont négocié une position de savoir. 


\section{La méthodologie d'analyse}

Nous situons nos propos dans le cadre général de l'analyse de discours ${ }^{5}$, un champ prolifique qui couvre une multitude d'approches très différentes qui varient en fonction des objets d'intérêt, du type de savoir qu'elles prétendent produire et des techniques qu'elles emploient. Comme le soulignent plusieurs auteurs dans leur effort de classification de ces approches, dont Maingueneau (1991) ou Taylor (2001), l'analyse de discours se prête autant à l'analyse de textes écrits qu'à l'analyse d'interactions verbales, et se déploie à la fois sur des registres linguistiques ou sémantiques. Le type d'analyse réalisé nous place dans le registre du sens issu des interactions verbales entre les participantes en tant que développement d'univers symboliques, comme le suggère la perspective interactionniste sur laquelle elle s'appuie.

De manière plus précise, notre manière d'aborder l'analyse du discours des participantes s'appuie sur un objet de recherche bien spécifique, celui des positions de savoir, objet qui guide le regard des analystes. Ces positions s'expriment le plus souvent sous deux aspects. D'une part, les enseignantes revendiquent une certaine conception d'un savoir issu d'une démarche de recherche et qui leur paraît un savoir pertinent pour la pratique; c'est là une manière pour elles d'orienter la démarche de groupe. D'autre part, elles expriment aussi leur manière de concevoir la complémentarité entre le rôle du chercheur et celui des praticiens dans cette démarche de recherche; c'est là une autre manière pour elles d'orienter la démarche de groupe. Dans l'analyse, nous recoupons ces deux angles pour identifier et caractériser les différentes positions de savoir chez les participantes.

Évoquons ici les concepts qui nous ont aidés à catégoriser le discours en vue d'identifier de manière opérationnelle les positions de savoir. Sur le plan discursif, leur engagement à revendiquer ces positions de savoir, sous l'un ou l'autre des deux angles, se traduit concrètement par ce que Davies et Harré (1990) nomment des «extensions indexicales" (indexical extension) et «typifiantes » (typification extension). Les « extensions indexicales » sont des séquences du discours qui montrent comment leur conception du savoir et de la complémentarité entre praticiens et chercheur est appuyée sur leurs expériences en contexte de pratique. Par exemple, lorsque l'une des enseignantes (participante 1: P1) rapporte comment elle a apprécié son expérience de formation avec un chercheur parce que ce dernier fournissait des procédures, elle déploie, ce faisant, sa conception du savoir. Les « extensions typifiantes » sont des séquences du discours qui montrent comment les enseignantes, pour mieux revendiquer leur conception du savoir et la complémentarité qu'elles attendent entre praticiens et chercheur, vont utiliser des typifications. Par 
exemple, lorsqu'une enseignante (participante 5 : P5) interpelle à plusieurs reprises la chercheuse pour obtenir d'elle la validation ou l'invalidation de ses pratiques, elle typifie son attente de complémentarité.

En terminant ce propos sur la méthodologie d'analyse, mentionnons qu'en cohérence avec l'interactionnisme symbolique, nous avons tenu compte de la dimension temporelle dans l'identification des positions de savoir des participantes. En effet, comme on le verra, nous avons d'abord cru bon de situer que l'affirmation des positions de savoir des participantes était déjà une réponse à l'offre de complémentarité proposée au départ par la chercheuse. Ensuite, nous avons distingué ce que nous avons appelé l'affirmation de la position de savoir de la négociation de sa viabilité, l'une étant, dans la logique de la négociation de la position, préalable à l'autre. Aussi, nous avons élargi notre analyse à des moments-types de négociation, moments qui s'échelonnent sur l'ensemble de la démarche d'investigation sur le terrain. Enfin, l'évocation d'un schéma intégrateur, artéfact matérialisant les processus de négociation, témoigne à sa façon de la dynamique coconstructive du savoir dans le temps.

\section{La présentation des résultats d'analyse des positions de savoir}

Avant d'éclairer le jeu des positions de savoir du groupe d'enseignantes, il nous faut rappeler la position de savoir de la chercheuse, car c'est à partir de cette position que les enseignantes se sont situées. C'est d'ailleurs ce à quoi nous convie Charaudeau (1995) qui soutient que les concepts de «positionnement » et de "négociation » doivent impérativement être liés au contrat de communication. En ce sens, dans le cas qui nous occupe ici, c'est la chercheuse qui leur fait une proposition de fonctionnement de groupe, suggérant ainsi une attente de complémentarité de la part des partenaires qui révèle du coup sa propre position. Sachant qu'elle sollicite l'explicitation des manières de faire l'évaluation formative des enseignantes, il se crée, lors des entretiens de groupe, une zone de partage qui donne lieu à des processus de négociation autour de ce qu'elles lient aux pratiques d'évaluation formative. En ce sens, dans cette démarche, elles identifient des dimensions qui seraient attachées à leurs manières de faire l'évaluation formative et en négocient par conséquent le territoire symbolique.

Les orientations dont témoigne ce format d'investigation reposent sur une certaine rationalité pratique dans laquelle s'inscrit la chercheuse, proche de la conception du savoir-agir en contexte du praticien chez Schön $(1983,1987)$, proche aussi de la «conscience pratique » de l'acteur social, tel que défini par Giddens (1987). Pour ce dernier, la conscience pratique se rapporte à tout ce que l'acteur sait faire pour se mouvoir dans la vie sociale, soit une sorte de savoir-faire qui repose sur un bagage de connaissances constitué des schèmes 
d'interprétation, soit des modes de représentation et de classification qui lui permettent d'agir et de rendre compte de ses raisons d'agir. Dans des contextes où est sollicitée la réflexivité de l'acteur, comme dans la démarche collaborative de recherche dont il est question ici, cette conscience pratique peut être rendue en partie discursive. C'est donc sur ces bases que la chercheuse a abordé les praticiennes, et on devine que cette rationalité pratique avancée auprès du groupe d'enseignantes comme démarche de coconstruction du savoir de recherche va induire un rapport de complémentarité dans la collaboration. En effet, s'inscrire dans cette rationalité pratique suppose une attente de mobilisation et d'explicitation de leurs manières de faire comme condition première de la construction du savoir chez les enseignantes. En corollaire, cela suppose, de la part de la chercheuse, une offre de facilitation à l'explicitation, voire de théorisation in situ des composantes qui émergent du collectif et qui traduisent le produit de leur négociation. On verra plus loin, à ce propos, le rôle joué par l'idée d'un schéma intégrateur des contributions des enseignantes comme levier de la coconstruction.

\section{L'affirmation d'une position de savoir et la négociation de sa viabilité}

Nous allons maintenant tenter de cerner de quelle manière chaque enseignante répond à l'offre de la chercheuse, soit son positionnement dans la démarche de coconstruction. Pour cela, nous établissons d'abord la position de savoir qu'affirme chaque enseignante, au départ de la démarche, en réponse à l'offre de la chercheuse. Nous établissons ensuite comment chaque enseignante, négociant un terrain de complémentarité, trouve une manière de rendre sa position de savoir viable dans la démarche proposée par la chercheuse ${ }^{6}$.

Le positionnement de la participante $1(\mathrm{P} 1)$

- L'affirmation d'une position de savoir "ce que je fais souvent, c'est que j'enseigne, je montre [je modélise], je vérifie avec l'ardoise..."

P1, au départ, ne semble pas à l'aise avec l'idée que le savoir va prendre sa source dans leurs pratiques, leurs manières de faire à elles, et que leur échange consistant à nommer, expliciter, voire théoriser ce qu'elles font suffira à statuer d'un savoir issu de la démarche de recherche. En ce sens, elle oppose à l'offre de la chercheuse une position de savoir différente. Elle s'annonce comme étant séquentielle et en arrive rapidement à identifier que ce qu'elle valorise comme savoir de pratique s'exprime en termes de procédures éprouvées, entre autres, pour mettre en œuvre l'évaluation formative. Elle laisse donc assez rapidement entendre que le savoir de recherche à coconstruire pour la pratique, dans la démarche, devrait prendre la forme de telles procédures éprouvées. En fait, on décode que, pour P1, la chercheuse devrait 
jouer le rôle de sélectionner, parmi les manières de faire qu'elles ont développées dans leur expérience, les procédures les plus éprouvées, celles qui sont jugées les plus efficaces. Ou encore que la chercheuse devrait proposer des procédures jugées efficaces et les enseignantes auraient alors le rôle de les valider dans leur pratique. L'analyse des propos tenus par cette enseignante nous indique qu'il y a sans doute un peu des deux, mais ce qui ressort surtout, c'est que le produit de la recherche devrait, pour elle, prendre la forme de procédures éprouvées, stabilisées, standardisées.

Il semble donc, par son insistance sur les procédures efficaces et standardisées, que P1 oppose à la rationalité pratique dans laquelle s'inscrit l'offre de la chercheuse, une rationalité davantage technique. Cela dit, elle n'évacue pas complètement cette rationalité pratique car, quand on analyse son propos, on remarque qu'elle reconnaît la nécessité que les enseignantes éprouvent les procédures suggérées par la chercheuse dans leur pratique. En effet, elle dit croire aux manières de faire développées dans l'expérience, à l'ajustement en situation, voire à l'improvisation. D'ailleurs, elle souligne que, pour elle, les procédures éprouvées ne viennent pas que des chercheurs, mais aussi de collègues enseignantes qui les développent et les testent dans le cadre de leur pratique, et auxquelles elle emprunte de telles procédures. Mais il n'en reste pas moins que, pour P1, comme on l'a illustré dans le paragraphe précédent, le savoir à coconstruire dans la démarche ici concernée doit prendre sa source chez la chercheuse qui pour elle doit jouer le rôle de sélectionner les procédures que la démarche aura permis de standardiser. C'est en cela qu'elle nous paraît s'inscrire davantage dans une rationalité technique. À maintes reprises, elle se réfère d'ailleurs à un chercheur qu'elle dit avoir apprécié, qu'elle appelle par ailleurs « son maître », et qui, précisément, lui avait proposé de telles procédures déjà standardisées par la recherche; celles-ci sont demeurées, pour elle, des procédures efficaces pour sa pratique, une sorte d'idéal de savoir de recherche pour la pratique.

- La négociation d'une viabilité à la position de savoir « tu répartis une tâche en petites tâches... et après ça tu évolues"

Compte tenu de l'offre de complémentarité proposée par la chercheuse, comment P1 s'ajuste-t-elle pour concilier sa position avec celle souhaitée dans le cadre de ce projet? L'analyse de ses propos nous amène à comprendre qu'elle n'attend pas que les procédures éprouvées viennent de la chercheuse, elle va plutôt puiser dans sa pratique quotidienne sa vision procédurale de l'évaluation formative; elle développera en ce sens l'idée d'une séquence formative. En effet, P1 explique comment elle s'y prend concrètement pour enseigner une notion et mettre en œuvre une évaluation formative de façon séquentielle. Elle débute habituellement par enseigner une leçon, étape par 
étape, en faisant réaliser des exercices aux élèves et en vérifiant leur compréhension régulièrement à l'aide de leur ardoise personnelle. Puis, elle leur propose un petit contrôle qui présente la notion décortiquée, afin de voir quels aspects précis de celle-ci ne sont pas consolidés par les élèves. Enfin, P1 fait faire aux élèves une série de tâches plus formelles tirées d'un matériel pédagogique la plupart du temps, jusqu'à ce que tout le monde soit au même niveau; pour les élèves qui présentent des difficultés lors de ces différentes étapes, elle adapte les tâches. En explicitant cette manière de mettre en œuvre l'évaluation formative, soit une alternance entre des façons standardisées et sériées de vérifier la compréhension des élèves et l'ajustement de ses interventions auprès d'eux, $\mathrm{P} 1$ est celle dans le groupe qui explicite des aspects plus formels de l'évaluation formative. C'est là l'originalité de sa contribution, tributaire de ce qu'on peut envisager comme sa manière de réconcilier rationalité pratique et rationalité technique.

Le positionnement de la participante 2 (P2)

- L'affirmation d'une position de savoir dans le groupe « J'ai l'impression qu'on déborde...j'essaie de suivre là! »

P2 n'est pas à l'aise non plus par rapport à l'offre de la chercheuse. L'image que la chercheuse leur renvoie, à l'effet que ce sont leurs manières de faire qui l'intéressent, voire que ce sont elles les expertes, ne va pas de soi pour elle. Bien qu'elle reconnaisse qu'une telle approche donne beaucoup d'ouverture aux enseignantes, leur permet de savoir que leurs pratiques ne sont pas jugées, elle demeure inquiète. En fait, elle exprime une attente que la chercheuse leur fournisse des balises pour ne pas que les discussions débordent de tous côtés, plus spécifiquement, qu'elle leur donne une définition de l'évaluation formative sur laquelle elles pourront toutes s'appuyer pour que, selon ses propos, sa propre conception de l'évaluation formative ne soit pas une fausse connaissance. "Que dit la littérature universitaire? », demande-t-elle. Dans les premières rencontres, elle manifeste même une impatience au fait que l'exploration des manières de faire l'évaluation formative les amène à explorer plusieurs chemins pour statuer des pratiques qui sont liées à l'évaluation formative : "ça va faire, dit-elle, je veux savoir c'est quoi! » Au fond, elle supporte mal que la définition vienne des enseignantes, qu'elle soit le produit de l'explicitation et de la théorisation in vivo de ce qu'elles considèrent être des pratiques d'évaluation formative. Mais c'est bien là le contrat que leur a proposé la chercheuse et avec lequel, on le verra, elle va composer compte tenu de sa position de savoir.

$\mathrm{P} 2$, comme P1, n'est pas contre le fait que, dans la démarche, on tienne compte des manières de faire des enseignantes, que ce soit dans les pratiques 
que s'incarnent les enjeux de l'évaluation formative. Et elle est prête à en témoigner. Ainsi, on ne peut pas dire qu'elle refuse complètement de s'inscrire dans une rationalité pratique, dans un savoir de recherche construit à partir des pratiques. Cependant, pour elle, l'explicitation des pratiques doit être canalisée par un cadre de référence, ce qu'elle appelle une définition commune de l'évaluation formative. À l'appui du cadre de référence censé livrer ce qu'elle conçoit comme la vraie connaissance, les manières de faire peuvent être apportées comme autant d'exemples de ce qui entre dans la définition. Et selon elle, c'est la chercheuse qui doit la donner cette définition. En ce sens, les pratiques à mobiliser devraient être illustratives et dépendantes du cadre de référence qui balise l'objet de savoir. C'est moins une rationalité technique qu'elle oppose à la rationalité pratique, qu'une rationalité théorique qu'elle met à l'avant-plan de la rationalité pratique. La définition abstraite ou intellectuelle précède, pour elle, la pratique concrète et contextuelle. La définition balise la pratique qui sert alors d'exemple illustratif de la définition qui est donnée de l'extérieur, une définition reconnue, une référence sur laquelle on peut appuyer la pratique.

- La négociation d'une viabilité à la position de savoir dans le groupe " J'avais besoin d'une base... j'ai travaillé avec le dictionnaire »

On devine que la chercheuse n'offre pas à P2 le cadre de référence attendu. Par contre, elle lui suggère de trouver ses propres balises pour parler de sa pratique et l'expliciter. Comment donc cette enseignante rend-elle viable sa position de savoir qui place une certaine rationalité théorique à l'avant-scène d'une rationalité plus pratique? C'est en prenant appui sur la définition fournie par un dictionnaire spécialisé en éducation que P2 se rassure, et en arrive ainsi à tirer de sa pratique des exemples de manières de faire l'évaluation formative. Selon ses dires, cette définition spécifique lui fait entre autres prendre conscience que l'évaluation formative non seulement permet aux élèves de recevoir une rétroaction sur leurs réalisations, mais permet aussi à l'enseignant d'ajuster ses interventions. Elle s'était oubliée là-dedans, dit-elle à l'issue de cet élargissement du regard à poser sur sa pratique que lui procure son nouveau cadre de référence. En fait, c'est cette définition qui lui procure une base sur laquelle construire, qui lui donne un point de référence pour amorcer une forme d'objectivation de sa pratique : "cette fois-là, je ne crois pas en avoir fait de l'évaluation formative ", précise-t-elle à l'occasion. Cette référence conceptuelle semble lui servir de guide pour identifier ce qui, dans ses pratiques explicitées, correspond à des manières de faire l'évaluation formative en classe, rendant du coup sa propre position de savoir plus opératoire, à l’intérieur de la rationalité pratique privilégiée dans la démarche de recherche. 
Le positionnement de la participante 3 (P3)

- L'affirmation d'une position de savoir dans le groupe «Les connaissances se construisent dans l'interaction! »

P3, contrairement aux deux autres, entre de plain-pied dans la proposition de complémentarité de la chercheuse. Si celle-ci manifeste, au départ, l'intention de miser sur leurs manières de faire l'évaluation formative, P3 s'attribue d'emblée cette expertise. Pour elle, c'est dans l'interaction avec les élèves et à partir de la tâche spécifique qu'on leur a proposée qu'on voit ce qui convient en termes de rétroaction et de soutien à l'apprentissage, en termes d'évaluation formative en acte. En ce sens, elle se dit très à l'écoute des élèves, de leurs besoins de soutien. Elle parle d'un flair que procure l'expérience de pratique. Il y a donc, pour elle, une pertinence à miser sur cette expérience acquise, ce flair, pour apporter un éclairage sur l'évaluation formative, ces manières de faire qu'elle a développées dans l'interaction et qu'elle a fait siennes. D'ailleurs, quand la chercheuse précise aux enseignantes que ses questions visent à dégager le sens de leur pratique, P3 se dit tout à fait en accord avec cette façon de faire. Et tout au long de la démarche, elle se montre particulièrement engagée dans l'explicitation, se faisant toujours très précise dans ses descriptions de pratiques de classe.

Cela dit, P3 conçoit que ses manières de faire ancrées dans l'expérience ne sont pas transmissibles à d'autres, suivant sa logique d'un savoir personnalisé lié à sa propre expérience de pratique : «tu peux avoir autant de méthodes que d'enseignantes ", dit-elle en ce sens. Si elle semble s'inscrire de plain-pied dans une rationalité pratique, à l'appui d'une conception très expérientielle d'un savoir ancré dans l'agir, et qu'ainsi sa position se concilie aisément avec l'offre de complémentarité de la chercheuse, elle laisse néanmoins un peu à la chercheuse le soin de composer avec le fait qu'il y ait autant de manières de faire que d'enseignantes. Faut-il arriver à définir des composantes communes à leurs pratiques respectives, viser un point de convergence dans le groupe? Elle ne semble pas s'en préoccuper outre mesure, tout occupée à valoriser sa propre expérience et celle des autres enseignantes. On verra qu'elle se distingue en cela de P4 qui s'inscrit pourtant comme elle dans une rationalité pratique.

- La négociation d'une viabilité à la position de savoir dans le groupe " J'ai essayé d'apporter selon mon enseignement »

Étant donné que la position de savoir de P3 semble s'harmoniser avec la proposition de la chercheuse, elle n'a pas vraiment à s'ajuster pour lui trouver une viabilité. Cependant, il est intéressant de regarder de manière plus spécifique comment elle répond à l'invitation de la chercheuse et contribue à la 
coconstruction d'un savoir sur les pratiques d'évaluation formative. D’emblée, il ressort que la rationalité pratique de cette enseignante s'exprime surtout à travers une vision très contextualisée de sa pratique d'évaluation. D’abord, P3 livre une description très fine et détaillée des exemples de pratiques d'évaluation formative qu'elle soumet au groupe, en termes de manières de faire, d'enjeux sous-jacents, et de prise en compte d'éléments contextuels. Également, elle explicite souvent les conceptions de l'apprentissage qui fondent ses pratiques, comme l'illustrent ces propos: "j'aime bien l'idée de déstabiliser au départ». La rationalité pratique chez P3 s'incarne aussi dans l'explicitation de ses manières de faire par l'expression d'une dynamique entre une routinisation - se donner un rituel hebdomadaire de pratique d'évaluation composé d'activités récurrentes - et l'improvisation - questionner les élèves selon les besoins interprétés dans l'interaction immédiate. Enfin, P3 commente sa pratique en expliquant comment l'évaluation formative se construit dans l'interaction lorsque, par exemple, elle encourage les élèves à se questionner eux-mêmes. Ces façons d'expliciter ses pratiques témoignent, pour elle, d'une conception très personnelle de la mise en ouvre l'évaluation formative, et permettent de comprendre pourquoi P3 soutient qu'il y a autant de méthodes que d'enseignantes.

Le positionnement de la participante 4 (P4)

- L'affirmation d'une position de savoir dans le groupe «C'est vraiment inscrit dans une démarche de réflexion! »

P4 témoigne d'une même valorisation de l'expérience que P3 et aussi d'une même confiance à l'égard d'un savoir de recherche qui se construit à partir des manières de faire situées des enseignantes. Elle exprime en ce sens la nécessité pour toute enseignante de s'ajuster en situation, de décoder les besoins des élèves, en termes de soutien à l'apprentissage. Assez proche du flair évoqué par P3, elle parle, à sa façon, d'un instinct qui permet d'improviser en classe. Elle entre donc elle aussi de plain-pied dans l'offre de complémentarité de la chercheuse qui vise précisément à expliciter ce flair et cet instinct dans la pratique de l'évaluation formative en classe, et cela, de manière très contextualisée. Comme $\mathrm{P}$, elle met aussi l'accent sur une évaluation formative qui se construit dans l'interaction et surtout sur la nécessité que les élèves se questionnent et réfléchissent sur leurs difficultés. Mais ce qui ressort de la contribution plus particulière de $\mathrm{P} 4$, c'est que cette visée de prise en charge de soi-même, d'autonomie, voire d'affranchissement chez les élèves, semble trouver un écho dans sa conception du savoir à coconstruire dans le groupe d'enseignantes. 
À la différence de P3 qui conçoit que l'expérience de chaque enseignante lui appartient et qu'elle est difficilement transmissible, P4 aborde la coconstruction avec un souci qu'à l'appui de l'explicitation des expériences de chacune, les enseignantes créent des ponts entre elles et se donnent une vision commune. En ce sens, et on le verra plus loin, c'est elle qui s'engage le plus intensément dans l'élaboration du schéma intégrateur des contributions du groupe. D’ailleurs, au terme de la démarche, et prenant appui sur cet artéfact, elle soutient que tout le monde a apporté beaucoup, voyant la coconstruction à travers la métaphore d'une boule de neige qu'on fait grossir, et revendiquant qu'il s'agit là d'une contribution collective. Plus que cela, et c'est là qu'on établit un lien entre sa visée d'affranchissement chez les élèves et sa position dans le groupe, P4 propose aux enseignantes de poursuive la démarche de réflexion et de questionnement autour d'autres thématiques liées à leurs pratiques; de surcroît, elle ajoute qu'elles peuvent désormais l'assumer sans l'aide de la chercheuse. En ce sens, on peut dire que $\mathrm{P} 4$ colore sa rationalité pratique d'une rationalité plus critique, au sens émancipatoire, à travers la reconnaissance de la force du groupe et surtout de son potentiel d'autonomie, voire d'affranchissement, dans le développement de son propre savoir.

- La négociation d'une viabilité à la position de savoir dans le groupe « Suite aux rencontres, on partait, et puis ça continuait de trotter [dans notre tête]...»

La position de savoir de P4, comme celle de P3, est en cohérence avec la rationalité pratique sollicitée par l'offre de complémentarité de la chercheuse. Ainsi, elle explicite elle aussi de manière détaillée ses manières de mettre en œuvre l'évaluation formative, soulevant au passage les enjeux qu'elle lie à ces pratiques : "ça questionne beaucoup, c'est lourd de conséquences », dit-elle en mettant également en relief la dimension très contextualisée de ces pratiques, l'ajustement in situ: "tu évalues constamment leur compréhension, tu réajustes constamment tes paramètres ». Qu'en est-il donc de sa contribution particulière à la démarche de coconstruction? En fait, la coloration plus critique de la rationalité pratique dans laquelle P4 s'inscrit s'apprécie de deux façons particulières. D'abord, l'explicitation de ses manières de faire semble représenter un levier pour réfléchir sur sa pratique, la questionner; par exemple, elle s'attarde aux types de questions qu'elle pose aux élèves et interprète que certaines donnent trop facilement les réponses [...] pour que ça soit vraiment formatif. $\mathrm{P} 4$ est également celle qui encourage ses collègues à questionner leurs pratiques: "ce n'est pas formatif », dit-elle à propos d'un exemple rapporté par $\mathrm{P} 1$, ou "à quelque part, je me dis que c'est notre faute ", répondant à cette même collègue qui déplore que les élèves n'ont pas le réflexe de se questionner par eux-mêmes. Par ailleurs, la discussion de groupe semble également être 
l'occasion pour elle de dépasser la seule explicitation des manières de faire. P4 est celle qui cherche à théoriser autour des pratiques de toutes les enseignantes au-delà de sa propre pratique, tentant de faire des recoupements, de dégager des composantes communes ou de nommer différentes enjeux qui seraient attachés aux pratiques d'évaluation formative. En effet, elle prend rapidement le leadership dans l'élaboration du schéma intégrateur des contributions des enseignantes, participant par là d'une façon très active à la conceptualisation du produit des délibérations, aux côtés de la chercheuse qui en assume la responsabilité explicite.

Le positionnement de la participante 5 (P5)

- L'affirmation d'une position de savoir dans le groupe «On ne le saura jamais, c'est quoi... qu'elle [la chercheuse] veut! »

Un peu comme P1 et P2, P5 manifeste son inconfort par rapport à la demande de complémentarité de la chercheuse qui s'inscrit, rappelons-le, dans une rationalité pratique. Encore là, l'idée d'expliciter ses manières de faire, sans plus de balises, semble l'inquiéter. Elle demande souvent à ses collègues, mais surtout à la chercheuse, de la valider, à savoir si les exemples de pratique qu'elle rapporte au groupe sont bel et bien des exemples d'évaluation formative en acte. "Suis-je dans le champ [sur la mauvaise voie]? » insiste-t-elle. Mais contrairement à P1 et P2 qui en viennent à exprimer leur façon de voir la démarche, l'une souhaitant que la démarche soit l'occasion de standardiser des procédures, l'autre de s'appuyer sur une définition commune, P5 ne laisse pas émerger de son discours une position de savoir affirmée qui ouvrirait à la négociation de celle-ci par rapport à l'offre de la chercheuse, comme on le voit chez les autres. Si ce n'est de se placer dans une position de réceptivité et d'attente de conformité par rapport à ce qui doit, pour elle, venir d'une demande bien spécifique de la chercheuse qui lui doit lui dire si c'est correct ou pas, si c'est ou pas de l'évaluation formative. En conséquence, elle se place la plupart du temps en position d'écoute plutôt que de prise de parole, laissant aux autres tout l'espace d'explicitation de leurs manières de faire. En outre, lorsque la chercheuse l'invite à expliciter ses propres façons de faire, elle a tendance à s'associer à la pratique d'une autre: "c'est la même chose ». Encore là, réaction de conformité, cette fois, à ses collègues.

Ce qui peut être interprété comme une difficulté à s'attribuer une compétence à rendre compte de ses manières de faire dans le groupe semble se transposer aussi dans une difficulté à s'attribuer une compétence à contribuer à la coconstruction du groupe, et ce, pour une raison difficile à cerner ici. Alors que les autres, de manière générale, discutent, font des distinctions entre les différentes versions des pratiques explicitées, formalisent, entre autres à travers 
le schéma intégrateur, des dimensions de l'évaluation formative, P5 se dit plus souvent qu'autrement mélangée par rapport aux propos de ses pairs. Il semble qu'il lui soit difficile de contribuer à l'élaboration du collectif, du moins difficile de s'attribuer une compétence comme contributrice au savoir. Dans quelle rationalité s'inscrit-elle? Difficile de le dire, sachant qu'elle ne revendique pas, au départ, une position de savoir affirmée, comme réponse à l'offre de complémentarité de la chercheuse, sinon une position de retrait, une position de non-savoir, si l'on peut dire. Si P5 contribue à la démarche de coconstruction du seul fait de son engagement jusqu'à la fin, on verra qu'elle participe activement malgré tout à la démarche de coconstruction d'un savoir sur les pratiques d'évaluation formative.

- La négociation d'une viabilité à la position de savoir dans le groupe « Je me disais : "Hé! c'est une bonne idée ça! »

Ce qui semble être une position de non-savoir en relation avec le contrat particulier proposé par la chercheuse n'exclut pas que P5 trouve le moyen de contribuer à sa façon à la coconstruction. En effet, son principal apport réside dans la reconnaissance de la compétence des autres dans leur effort d'explicitation du sens qu'elles accordent à l'évaluation formative. En effet, elle met fréquemment en valeur les idées émises par ses collègues - «c'est bon ce que tu viens de dire!", dit-elle par exemple - ou encore l'expertise particulière qu'elle accorde à l'une d'elles : «tu as travaillé fort pour ces élèves-là [ceux dits en difficulté d'apprentissage] ». En ce sens, elle semble s'inscrire dans le mouvement de la rationalité pratique en encourageant l'explicitation des façons de faire de ses pairs, voire en la facilitant par valorisation. Et ce qui vaut pour elles vaut aussi pour la chercheuse. De fait, P5 se dit impressionnée par le travail de théorisation in situ effectué par la chercheuse symbolisé par le schéma. Par ailleurs, il est intéressant d'établir un lien entre cette compétence qu'elle reconnaît aux membres du groupe et qui semble faire sa couleur particulière dans la démarche de coconstruction, et ses manières de faire l'évaluation formative, du moins, pour le peu qu'elle en explicite dans le cadre de la démarche de recherche. En effet, le mode d'intervention en évaluation formative qu'elle rapporte semble surtout reconnaître aux élèves une compétence à se donner mutuellement une rétroaction formative. Elle privilégie, de manière plus sensible, une aide apportée par les élèves eux-mêmes, par opposition à la seule intervention de l'enseignant : "ça peut être bon de se le faire dire par quelqu'un d'autre [que l'enseignant] », dit-elle, en ce sens. Ainsi, la stratégie pédagogique qu'elle privilégie en classe, avec son groupe d'élèves, et le type de participation qu'elle met en scène, avec le groupe d'enseignantes, semblent analogues. 


\section{Des moments-types de négociation des positions et le rôle du schéma intégrateur}

En suggérant que chaque enseignante négocie une viabilité à sa position de savoir pour tenir compte de l'offre de position suggérée au groupe par la chercheuse, on risque de croire qu'à partir de là, la complémentarité entre la chercheuse et les participantes, et entre les participantes elles-mêmes, est assurée. Loin de là! D’une part, on pourrait dire que les positions de savoir se négocient tout au long de la démarche, dépendant des enjeux de la discussion et de la manière dont les participantes, à un moment ou à un autre, s'y sentent engagées ou interpellées par rapport à leur conception du savoir à coconstruire, par rapport aussi aux pratiques diversifiées qu'elles mettent en scène dans la démarche d'explicitation. D'autre part, soulignons que la démarche ne vise pas une sorte de consensus plat qui ferait que toutes partageraient une seule et même conception à propos de l'évaluation formative, une pensée unique qui glisserait vers une pratique unique. La démarche de coconstruction, telle que proposée par la chercheuse dans la perspective d'une rationalité pratique, encourage plutôt, en allant chercher les différentes manières de faire, en contexte, l'expression d'une complexité de l'agir qui ne peut se passer des nuances, des distinctions, voire des débats autour de leurs pratiques. En ce sens, la démarche ainsi aménagée encourage une négociation des positions, et cela, jusqu’à la fin du processus.

Pour en rendre compte, nous présentons, à titre d'illustration, quelques moments-types où se négocient, d'une manière ou d'une autre, les positions de savoir. Pour ce faire, nous évoquons trois de ces moments, l'un dit de confrontation, l'autre de suspension, enfin, un autre d'harmonisation des positions. Bien sûr, il ne s'agit pas d'une catégorisation exhaustive, mais bien d'une illustration, parmi un éventail de possibles, de la diversité des moments où se négocient les positions de savoir des enseignantes, et qui sont contributifs à la démarche de coconstruction d'un savoir sur les pratiques d'évaluation formative. Par ailleurs, inutile de nier qu'au fil de la démarche, au-delà de ces moments fugaces ainsi qualifiés, les positions différenciées en viennent à converger, à s'allier, somme toute, dans le sens d'une coconstruction de savoir partagé. Nous sommes en mesure de rendre compte aussi de ce mouvement de convergence des positions à travers le schéma intégrateur autour duquel se rallient les participantes, et qui leur servira, pourrait-on dire, de symbole, d'artéfact, voire d'outil conciliateur de leurs positions de savoir respectives.

\section{L'exemple d'un moment de confrontation}

Nous appelons un moment de confrontation une séquence d'interactions pendant laquelle, dans le cours de l'échange, les participants mobilisent leurs 
positions respectives dans un mouvement d'opposition. Un tel moment, qui implique de manière particulière $\mathrm{P} 1$ et $\mathrm{P} 4$, est identifié lorsque ces dernières discutent de leurs manières respectives de mettre en œuvre l'évaluation formative en s'appuyant sur un exemple précis, soit l'enseignement des fractions. Alors que P1 propose une démarche étapiste et standardisée pour montrer aux élèves comment réaliser des opérations entre fractions, $\mathrm{P} 4$ réagit vivement en soutenant que l'important est qu'ils soient plutôt capables de comprendre le sens de la fraction, de le représenter, mais pas techniquement parlant. Pour cette dernière, le but n'est pas d'amener les élèves à réaliser un exercice, mais de bien saisir, de comprendre le sens de la fraction. Cette réponse de $\mathrm{P} 4$ à $\mathrm{P} 1$, qui est d'ailleurs accueillie favorablement par l'ensemble des enseignantes, illustre que les différents types de rationalité mis en scène entrent parfois en collision lors des négociations sur les pratiques d'évaluation formative, dans ce cas précis, la friction étant tributaire de l'opposition entre une rationalité plus technique, celle de P1 à travers sa vision procédurale, et une plus pratique, celle de $\mathrm{P} 4$, qui valorise le sens construit en contexte et dans l'interaction avec les élèves.

\section{L'exemple d'un moment de suspension}

Nous appelons un moment de suspension une séquence d'interactions pendant laquelle, dans le cours de l'échange, les participants mobilisent leurs positions respectives dans un mouvement de retranchement. L'un de ces moments s'amorce alors que prend place une discussion autour de l'identification d'épisodes d'évaluation formative dans les pratiques respectives de P1, P3 et $\mathrm{P} 4$, cette dernière souhaitant qu'elles se donnent des balises communes pour les aider dans cette tâche. Rapidement, P1 et P3 se positionnent contre cette proposition, mais pour des raisons qui paraissent différentes. En ce qui concerne P1, dont la vision de la mise en œuvre de l'évaluation formative semble plutôt procédurale, comme nous l'avons fait valoir précédemment, elle affirme qu'elle sait identifier ces moments en s'appuyant sur ce qu'elle a retenu des enseignements d'un chercheur - son maître - soit des épisodes d'évaluation formative où elle administre une série de tâches formalisées sur papier qui lui permettent d'ajuster son enseignement. Malgré la proposition de P4, P1 se retranche dans sa position: "mais moi je n'ai pas encore changé mon idée », dit-elle. Quant à P3, en toute logique avec sa position de savoir qui affirme l'expérience de sa propre pratique, elle ne semble pas prendre en considération la proposition de P4 en soutenant qu'elle sait identifier les épisodes d'évaluation formative dans sa pratique : «je sais ce que je vais aller chercher ", soutient-elle à deux reprises. En somme, tout semble indiquer que les partenaires, lors de l'échange, ont tendance revenir à leurs positions respectives. 


\section{L'exemple d'un moment d'harmonisation}

Nous appelons un moment d'harmonisation une séquence d'interactions pendant laquelle, dans le cours de l'échange, les participantes mobilisent leurs positions respectives dans un mouvement de convergence. L'un de ces moments, qui implique de manière particulière P4 et P3, est identifié alors que la discussion porte sur le type de questions à poser dans l'interaction aux élèves pour soutenir leurs apprentissages. P4 soutient alors que le choix des questions à poser relève d'un certain art qui se déploie en contexte. P3, semblant abonder en ce sens, parle d'une forme d'improvisation, reposant sur l'expérience, laquelle permettrait de savoir quels types de questions seraient susceptibles de provoquer l'impact recherché chez les élèves dans un contexte donné. Dans cet exemple, les enseignantes se confortent l'une l'autre dans leur conception très contextuelle ou très expérientielle de l'évaluation formative, comme dans un mouvement de consolidation ou de légitimation mutuelle de leur rationalité pratique partagée. Cela dit, à d'autres moments d'échanges, la position plus émancipatoire de $\mathrm{P} 4$, qui la porte à vouloir que le groupe dépasse les manières de faire respectives et se donne une vision partagée de l'évaluation, entre en confrontation avec la position de P3 qui demeure toujours centrée sur une vision plus idiosyncrasique de l'évaluation, propre à chaque enseignante.

\section{À propos du schéma intégrateur}

Tel que mentionné précédemment, au fil des négociations, les enseignantes, guidées par la chercheuse, ont cheminé vers la construction d'une représentation de leurs pratiques d'évaluation formative qui saurait rallier leurs différentes positions. Cette représentation prend la forme d'un schéma proposé à l'initiative de la chercheuse qui y voit d'abord un moyen de soumettre aux participantes une interprétation de leurs propos en cours de démarche à des fins de validation, proposant du coup un artéfact aménageable sur lequel prendre appui pour négocier autour des pratiques d'évaluation formative ${ }^{7}$. Mais en quoi, pour chacune, ce schéma est-il un symbole d'une réconciliation possible des positions de savoir? Chez P4, qui, rappelons-le, prend le leadership de son élaboration, le schéma paraît représenter une façon de s'affranchir, de s'émanciper, en proposant une représentation d'une compréhension transversale à toutes les enseignantes de ce que seraient les pratiques d'évaluation formative. En y voyant un outil diffusable, transférable à d'autres enseignantes et enseignants, il semble satisfaire pour elle sa rationalité critique qui la porte dans un mouvement d'autonomisation. C'est justement en cela que le rapport au schéma de P4 semble se distinguer de celui qu'entretient P3 à l'égard de ce même symbole. En effet, celle-ci se dit épatée devant les différentes versions du schéma, comme si elles s'inscrivaient dans la continuité 
théorisée des pratiques telles qu'elle les conçoit. On se rappelle que pour P3 qui a une vision très contextualisée des pratiques, il y a autant de façons de faire que d'enseignantes, et par extension, il pourrait y avoir autant de schémas que de groupes d'enseignants se penchant sur le même objet. Par ailleurs, si les positions de P1 et de P2 sont également ralliées au schéma, on peut penser que c'est pour d'autres raisons. Pour elles, il semble en effet constituer le référent extérieur qu'elles attendaient. Cependant, en cohérence avec leurs positions respectives, le schéma aurait le sens d'une représentation de pratiques éprouvées pour P1, et d'une définition [enfin] stabilisée pour P2. Finalement, on ne s'étonne pas d'apprendre que $\mathrm{P} 5$ se dit très impressionnée par le schéma. On peut interpréter sa réaction comme une certaine satisfaction à l'endroit de cette représentation symbolique qui lui renvoie une image de compétence comme participante, alors qu'elle-même ne semble pas s'en attribuer. Néanmoins, en cohérence avec sa position de non savoir, elle ne s'approprie pas le schéma comme les autres semblent le faire. En effet, à propos des négociations autour d'un élément qui y est annoté, elle dit à ses collègues : «de toutes façons, elle [la chercheuse] va faire ce qu'elle veut avec [ce schéma] », renvoyant par là même la propriété de cet artéfact à la chercheuse. En somme, même si les enseignantes ne semblent pas y accorder la même signification, le schéma est devenu, au fil des entretiens, le catalyseur d'une convergence des positions, facilitant l'ajustement de positions de savoir distinctes ${ }^{8}$.

\section{Discussion et conclusion autour du jeu des positions de savoir}

Il est intéressant de regarder l'analyse dont nous venons de rendre compte sous l'angle de la complémentarité des contributions des partenaires dans une approche collaborative en éducation. Ce modèle s'inscrit dans une problématique où l'adéquation entre champ de recherche et champ de pratique, comme le souligne Barbier (2001), est considéré comme un enjeu majeur pour le développement d'une profession et plus spécifiquement pour la profession enseignante. Pour mieux assumer cet enjeu, et à l'instar d'autres approches de recherches dites participatives, l'approche collaborative propose une contribution des praticiens à la démarche de recherche. On traduit le plus souvent cette participation souhaitée comme une « ouverture » du chercheur au point de vue des praticiens sans ouvrir plus avant cette boîte noire. Notre analyse suggère que cette ouverture est en soi une prise de position par rapport au savoir à coconstruire. Dans la recherche ici concernée, l'ouverture de la chercheuse est tributaire de sa posture compréhensive ainsi que de sa conception des enseignants en tant qu'acteurs compétents (Giddens, 1987); en soi, cette ouverture est déjà une prise de position qui oriente le déroulement de l'investigation en suggérant que les enseignantes s'engagent, sous sa 
facilitation, à expliciter leurs manières de faire, à en négocier la validité entre elles, et à en offrir une théorisation plus ou moins formelle. En ce sens, cette ouverture est donc au cœur d'une tension entre un souci de rigueur pour toute démarche méthodologique, en recherche interprétative, et la nécessité de maintenir une certaine souplesse pour mieux "sentir» le terrain, comme le suggère Cyrulnik (2003) qui invite les chercheurs à laisser leur " autoroute " pour emprunter les « sentiers de chèvres ».

Face à cette ouverture, on a vu que les participantes ne sont pas naïves. En effet, le regard interactionniste que nous avons posé sur la démarche laisse à penser qu'elles comprennent très vite elles-mêmes que cette ouverture est une position de savoir bien affirmée de la chercheuse, une position qui se traduit par une offre de complémentarité qu'elles ne sont pas sûres de vouloir assumer comme telle, du moins pas sans la négocier en relation avec leur propre position de savoir. Et ce n'est pas parce que la position de la chercheuse fait en sorte qu'elle valorise leur point de vue de praticiennes et qu'elle souhaite que ce point de vue soit mis à l'avant-plan de la démarche que cela suffit pour qu'elles y adhèrent. Bien au contraire, certaines voient autrement le rapport de complémentarité et souhaitent au départ mettre à l'avant-plan le point de vue de la chercheuse (on pense, par exemple, à P2 qui souhaite une définition de l'évaluation formative). Par ailleurs, ce que notre analyse met en relief, c'est qu'elles s'inscrivent tout de même dans l'orientation proposée par la chercheuse, au meilleur bénéfice de la démarche de coconstruction. Cependant, cet engagement ne se fait pas au détriment de leur propre position de savoir qu'elles rendent viable, c'est-à-dire qu'elles la concilient avec celle de la chercheuse pour répondre à son offre de complémentarité et la coordonnent avec celle des autres pour contribuer à la coconstruction. En ce sens, la complémentarité entre chercheur et praticiens est un produit négocié à partir des positions de savoir de chacun.

Reste la question du statut que chacun accorde à son propre savoir et au savoir de l'autre. Car dans la notion de position de savoir avancée par Darré (1999), il y a cette idée qu'un savoir est avant tout une prétention de savoir, une capacité d' «intimider » l'autre et de lui faire sentir que «moi je sais » et que « lui sait moins ». En d'autres mots, à l'appui de l'œuvre de Foucault, on dirait que le savoir est un pouvoir qu'un acteur mobilise et fait valoir auprès d'un autre, dans un contexte d'interaction donné. Là encore, la perspective interactionniste nous rattrape... Quelles en sont les implications pour notre analyse? Il est intéressant de voir le jeu des positions de savoir à travers ce pouvoir que les unes et les autres s'attribuent dans la démarche. Quand on dit que P3 entre dans l'offre de complémentarité proposée par la chercheuse en assumant ses manières de faire, en les explicitant sans sembler craindre le 
jugement des autres, c'est qu'elle s'attribue un pouvoir de contribuer au savoir de recherche, sur la base d'un savoir de pratique qu'elle est fière de mettre en scène. On a vu que $\mathrm{P} 4$ va plus loin en ramenant tout le pouvoir du savoir du côté des praticiennes lorsqu'elle soutient, à la fin de la démarche, qu'il serait intéressant de faire une démarche semblable autour d'une autre thématique, mais cette fois sans la chercheuse. Le pouvoir du groupe de praticiennes dans la production du savoir devient ici manifeste. On a vu aussi que P2 et P1 remettent à la chercheuse un pouvoir plus grand dans le savoir de recherche à produire, en s'inscrivant dans un certain rapport de dépendance, pour ne pas dire de soumission, du moins en hiérarchisant les positions. Pour ces enseignantes, le savoir doit venir comme une prescription de la chercheuse et leur rôle de praticiennes, dans la démarche, devrait en être un d'application, de validation, voire d'illustration d'un savoir venu du monde de la chercheuse. Quant à P5, on a vu que pour elle, le savoir est surtout l'affaire de ses partenaires, ayant tendance à s'attribuer une position de non-savoir ou de savoir par procuration de ce que les autres proposent et que, cela dit, elle valorise, contribuant ainsi à sa manière à la démarche de coconstruction. En somme, le jeu des positions de savoir concerne aussi une manière d'accorder à soi et à l'autre un pouvoir de contribution à la coconstruction du savoir, incluant l'établissement d'un rapport plus ou moins égalitariste ou hiérarchique, plus ou moins assujettissant ou affranchissant.

L'éclairage que nous venons d'esquisser sur le jeu des positions de savoir dans le cadre de cette recherche collaborative portant sur les manières de faire l'évaluation formative ouvre sur toute la complexité inhérente au rapport de coconstruction qui s'établit, dans une telle approche, entre le chercheur et les praticiens. Il soulève du même coup un questionnement élargi sur toute pratique d'enquête qualitative, entretiens individuels ou de groupe, où s'engage toujours un contrat de communication, et la nécessité d'en examiner les conditions de réalisation pour mieux en cerner les enjeux. C'est à ce prix que s'exerce, en recherche, une vigilance méthodologique, que d'aucuns appellent aussi une réflexivité méthodologique (Demazière, 2008)... à laquelle cet article souhaite contribuer.

\section{Notes}

${ }^{1}$ Les auteurs remercient le Conseil de recherche en sciences humaines du Canada (CRSH) dont le financement, sous la forme d'une bourse d'excellence, a rendu possible la réalisation de la thèse à partir de laquelle est tiré cet article.

${ }^{2}$ Les lecteurs se référeront à la thèse pour connaître tous les détails sur le format d'investigation (Morrissette, 2009), notamment concernant les deux autres types 
d'activités, attachés à des entretiens individuels, qui ont caractérisé la démarche sur le terrain.

${ }^{3}$ Comme le soulignent Davies et Harré (1990), le terme de « position » se prête bien à l'étude d'un phénomène dynamique tel les interactions d'un groupe d'acteurs.

${ }^{4}$ La relation plus égalitariste entre chercheurs et praticiens à laquelle invite Darré (1999) peut être rapprochée du type de relation entre chercheurs et citoyens prôné par Callon, Lascoumes et Barthe (2001) qui proposent d'intégrer le point de vue « averti » des citoyens dans les pratiques scientifiques.

${ }^{5}$ À titre informatif, mentionnons que cette analyse de discours est réalisée à partir du matériau issu de cinq entretiens de groupe d'environ 2 h 30 chacun qui ont constitué les données originales d'une recherche doctorale interprétative portant sur les manières de faire l'évaluation formative des apprentissages selon un groupe de cinq enseignantes du primaire, volontaires, choisies par leurs pairs (Morrissette, 2009).

${ }^{6}$ Dans les sections qui suivent, les mots en italique sont ceux des enseignantes.

${ }^{7}$ Plusieurs chercheurs liant leurs travaux à l'interactionnisme symbolique tels que Woods (1992) et d'autres s'intéressant à l'analyse en groupe tels que Campenhoudt, Chaumont et Franssen (2005) reconnaissent l'intérêt d'un processus de validation continu en cours d'investigation permettant au chercheur de tester ses interprétations. La modalité retenue dans le cadre de notre recherche est un schéma représentant les différentes contributions des enseignantes que les lecteurs pourront consulter sous sa forme finalisée dans la thèse de Morrissette (2009).

${ }^{8}$ Le schéma illustre bien, nous semble-t-il, l’idée d' « accord pragmatique » chez Pépin (1994). En effet, s'il fait consensus, il rejoint chacune différemment dans sa position de savoir respective. Autrement dit, il est l'outil commun par lequel chacune trouve une viabilité à sa position de savoir; il constitue une «zone carrefour » des viabilités. En outre, on peut penser que cette zone permet une coordination des contributions vers un produit collectif, l'aboutissement de la démarche de coconstruction de savoir.

\section{Références}

Allal, L. (1991). Stratégies d'évaluation formative: conceptions psychopédagogiques et modalités d'application. Dans L. Allal, J. Cardinet, \& P. Perrenoud (Éds), L'évaluation formative dans un enseignement différencié (6 éd.) (pp. 153-183). Berne, CH : Peter Lang.

Barbier, J.-M. (2001). La constitution de champs de pratiques en champs de recherches. Dans J.-M. Baudoin, \& J. Freidrich (Éds), Théories de l'action et éducation (pp. 305-317). Bruxelles : De Boeck.

Becker, H.S. (1986). Doing things together. Evanston, IL : Northwestern University Press. 
Becker, H.S. (2004). Quelques idées sur l'interaction. Dans A. Blanc, \& A. Pessin (Éds), L'art du terrain. Mélanges offerts à Howard S. Becker (pp. 245-265). Paris : L’Harmattan.

Becker, H.S., \& McCall, M.M. (Éds.). (1990). Symbolic interaction and cultural studies. Chicago : University of Chicago Press.

Bednarz, N., Desgagné, S., Diallo, P., \& Poirier, L. (2001). L’approche collaborative de recherche: une illustration en didactique des mathématiques. Dans P. Jonnaert, \& S. Laurin (Éds), Les didactiques des disciplines. Un débat contemporain (pp. 177-207). Québec: Presses de l’Université du Québec.

Bell, B., \& Cowie, B. (2001). Formative assessment and science education. Dordrecht, NL : Kluwer Academic Publishers.

Callon, M., Lascoumes, P., \& Barthe, P. (2001). Agir dans un monde incertain : essai sur la démocratie technique. Paris : Seuil.

Campenhoudt, L. van, Chaumont, J.-M., \& Franssen, A. (2005). La méthode d'analyse en groupe. Application aux phénomènes sociaux. Paris : Dunod.

Certeau, M. de (1990). L'invention au quotidien (vol. 1). Paris : Gallimard.

Charaudeau, P. (1995). Le dialogue dans un modèle de discours. Cahiers de linguistique française, 17, 141-178.

Couture, C. (2002). Étude du processus de co-construction d'une intervention en sciences de la nature au primaire par une collaboration praticienchercheur. Thèse de doctorat inédite, Université du Québec à Chicoutimi, Québec.

Cyrulnik, B. (2003). Les sentiers de chèvres et l'autoroute ou le premier temps de l'éthique. Dans V. Duclert, \& A. Chatriot (Éds), Quel avenir pour la recherche? Cinquante savants s'engagent (pp. 70-79). Paris : Flamarion.

Darré, J.-P. (1999). La production de connaissance pour l'action. Arguments contre le racisme de l'intelligence. Paris: Éditions de la Maison des sciences de l'homme et Institut National de la Recherche Agronomique.

Davies, B., \& Harré, R. (1990). Positioning: The discursive production of selves. Journal for the theory of social behaviour, 20(1), 43-63.

Demazière, D. (2008). L'entretien biographique comme interaction. Négociations, contre-interprétations, ajustement de sens. Langage et Société, 123, 15-35. 
Desgagné, S. (1997). Le concept de recherche collaborative : l'idée d'un rapprochement entre chercheurs universitaires et praticiens enseignants. Revue des sciences de l'éducation, 23(2), 371-393.

Desgagné, S. (2001). La recherche collaborative: nouvelle dynamique de recherche en éducation. Dans $\mathrm{M}$. Anadón (Éd.), Nouvelles dynamiques de recherche en éducation (pp. 51-76). Québec: Presses de l'Université Laval.

Desgagné, S. (2007). Le défi de coproduction de savoir en recherche collaborative : autour d'une démarche de reconstruction et d'analyse de récits de pratique enseignante. Dans $\mathrm{M}$. Anadon (Éd.), La recherche participative: multiples regards (pp. 89-121). Québec: Presses de l’Université du Québec.

Desgagné, S. Bednarz, N., Couture, C. Poirier, L., \& Lebuis, P. (2001). L'approche collaborative de recherche en éducation : un nouveau rapport à établir entre recherche et formation. Revue des sciences de l'éducation, 27(1), 33-64.

Elliott, S.M. (1999). Teacher formative assessment : influences and practice case study research at the year one level. Thèse de doctorat inédite, University of London.

Giddens, A. (1987). La constitution de la société. Paris : Presses universitaires de France.

Goffman, E. (1973). La mise en scène de la vie quotidienne. Paris : Minuit.

Guignon, S., \& Morrissette, J. (2006). Quand les acteurs mettent en mots leur expérience. Recherches qualitatives, 26(2), 19-36.

Maingueneau, D. (1991). L'Analyse du Discours. Introduction aux lectures de l'archive. Paris : Hachette Supérieur.

Morrissette, J. (2002). Évolution de la conception de l'évaluation formative des apprentissages à travers le discours ministériel depuis 1981. Mémoire de maîtrise inédit, Université du Québec en Abitibi-Témiscamingue, Québec.

Morrissette, J. (2009). Manières de faire l'évaluation formative des apprentissages selon un groupe d'enseignantes du primaire: une perspective interactionniste. Thèse de doctorat inédite, Université Laval, Québec.

Morrissette, J., \& Maheux, G. (2007). Évolution de la conception de l'évaluation formative des apprentissages à travers le discours ministériel québécois depuis 1981. Revue des sciences de l'éducation, 33(3), 727-748. 
Pépin, Y. (1994). Savoirs pratiques et savoirs scolaires : une représentation constructiviste de l'éducation. Revue des sciences de l'éducation, 20(1), 63-85.

Perrenoud, P. (1998). L'évaluation des élèves. De la fabrication de l'excellence à la régulation des apprentissages. Entre deux logiques. Bruxelles : De Boeck.

Scallon, G. (2000). L'évaluation formative. Québec : Renouveau Pédagogique.

Schön, D.A. (1983). The reflective practitioner. New York : Basic Books.

Schön, D.A. (1987). Educating the reflective practitioner. San Franscico: Jossey Bass.

Taylor, S. (2001). Locating and conducting discourse analytic research. Dans M. Wetherell, S. Taylor, \& S. Yates (Éds), Discourse as data : A guide for analysis (pp. 5-48). London : Sage.

Woods, P. (1992). Symbolic interactionnism : Theory and method. Dans M.D. Le Compte, W. L. Millroy, \& J. Preissle (Éds), The handbook of qualitative research (pp. 337-404). New York : Academic Press.

Joëlle Morrissette est professeure adjointe à la Faculté des sciences de l'éducation de l'Université de Montréal. Ses travaux s'inscrivent dans les domaines de l'évaluation des apprentissages, de l'interactionnisme symbolique ainsi des savoirs pratiques, et portent la marque d'une préoccupation particulière pour l'épistémologie de la recherche. Sur le plan méthodologique, elle privilégie les investigations collaboratives sur le terrain par le biais d'entretiens individuels et de groupe avec les enseignantes et les enseignants. Sur le plan analytique, elle aborde ses objets d'étude sous l'angle des interactions qui lient les acteurs de la situation éducative au quotidien, cherchant à rendre compte des significations qu'ils engagent dans ces interactions. À cette fin, et concevant que le sens est indissociable de la manière dont il est produit, elle recourt à des analyses de discours, plus précisément à l'analyse de conversations.

Serge Desgagné est professeur titulaire à la faculté des sciences de l'éducation de l'Université Laval. Il oeuvre à la formation des enseignantes et enseignants, initiale et continue, plus particulièrement dans le champ de l'intervention et de la relation pédagogiques, avec un intérêt particulier pour les modes d'intégration théorie et pratique. Ses travaux de recherche et ses publications portent, d'une part, sur la formalisation des savoirs d'action et d'expérience des enseignants, entre autres, par l'utilisation de récits de pratique et, d'autre part, sur le développement de l'approche de recherche collaborative en éducation. 